科 学 通 报

\title{
五倍子酸和酯其自由基的稳定性及 分子内氢键相互作用*
}

\author{
严继民 \\ (中国科学院化学研究所, 北京 100080)
}

\author{
颜达予 \\ (中国科学技术大学研究生院, 北京 100041)
}

\section{关键词五倍子酸、五倍子酸醋、自由基的稳定性、分子内虫键、MNDO}

在五倍子和茶叶中普遍存在五倍子酸及其烷基和茶基酯, 它们具有很好的抗氧化性, 防腐 性及收玫性等作用, 被广泛用于油脂、染料、纺织、冶金、石油、化工、食品及医药等工业. 经研 究, 认为它们的这些活性与其极易形成自由基的性质有关 ${ }^{[1-4]}$. 对自由基的结构及其能够稳 定存在的原因至今研究的还不多, 只是凭经验认定是羭基对位的羟基失氢形成自由基. 在另 一篇论文中 ${ }^{1}$, 我们计算了各种可能生成的自由基的生成热, 从能量的观点指出, 五倍子酸及 其酯的羧基对位的羟基的确最易失氢形成自由基.

本文进一步计算及分析它们的电子结构, 从电子结构和氢键的观点来讨论五倍子酸及其 酷容易生成稳定自由基的原因,并且给出结构的定量的数据.

\section{1 方 法 和 模 型}

用量子化学 MNDO/UHF 方法, 对五倍子酸 (Gallic Acid, GA) 及其甲酯 (MG), 乙酯 $(E G)$, 丙酯 (PG), 异丙酯 (iso-PG), 丁酯 (BG) 和叔丁酯 (t-BG), 以及所有这些分子的羧基对 位和间位羟基失氢形成的自由基进行了计算. 计算时对所有分子及其自由基的键长, 键角和 二面角几何内坐标参数进行全面的优化, 优化时球优化误差指数 IEND 取最高精度 4.

\section{2 结 果 和 讨 论}

对所有上述这些分子及其自由基计算的结果表明, 总起来看, 这些分子及其自由基在内部 可以分为两部分, 一部分为取代苯基 $(\mathrm{HO}){ }_{3} \mathrm{C}_{6} \mathrm{H}_{2}$. 共一平面, 另一部分为取代羧基. $\mathrm{COOR}$ 也 共一平面, 并且两个平面互相垂直, 如图 1 所示.

比较酸和酯的结构差别, 其基本骨架的结构参数没有什么变化, 所变化的仅仅是取代基 $\mathrm{R}$; 比较分子和自由基的差别, 其羧基部分的结构参数没有什么变化, 所变化的仅仅是取代苯基 部分的结构参数. 本文感兴趣的是五倍子酸及酯自由基化前后结构参数的变化. 表 1 给出这 部分数据.

从表 1 中原子间距可看出, 五倍子酸及其酯自由基化后有利于邻近羟基间形成分子内氢

1993-07-13 收稿, 1993-11-08 收修改稿.

* 国家自然科学基金资助项目.

1) 颜达予等,物理化学学报, 待发表. 
表 1 五倍子酸及其酯自由基化前后有关各原子间距离的变化 $(\AA)$

\begin{tabular}{llcc}
\hline \multicolumn{1}{c}{ 原子对 } & 分子的 & 对位自由基的 & 间位自由基的 \\
\hline $\mathrm{H}(1)-\mathrm{O}(1)$ & 0.9515 & - & 0.9487 \\
$\mathrm{H}(2)-\mathrm{O}(2)$ & 0.9482 & 0.9486 & - \\
$\mathrm{H}\left(2^{\prime}\right)-\mathrm{O}\left(2^{\prime}\right)$ & 0.9480 & 0.9486 & 0.9491 \\
$\mathrm{H}(1)-\mathrm{O}(2)$ & 3.1921 & - & 2.5388 \\
$\mathrm{H}(1)-\mathrm{O}\left(2^{\prime}\right)$ & 3.2335 & - & 3.7307 \\
$\mathrm{H}(2)-\mathrm{O}(1)$ & 2.5628 & 2.5023 & - \\
$\mathrm{H}\left(2^{\prime}\right)-\mathrm{O}(1)$ & 2.5431 & 2.5048 & 2.4110 \\
$\mathrm{O}(1)-\mathrm{C}(1)$ & 1.3644 & 1.2392 & 1.3470 \\
$\mathrm{O}(2)-\mathrm{C}(2)$ & 1.3537 & 1.3456 & 1.2987 \\
$\mathrm{O}\left(2^{\prime}\right)-\mathrm{C}\left(2^{\prime}\right)$ & 1.3537 & 1.3456 & 1.2987 \\
$\mathrm{C}(1)-\mathrm{C}(2)$ & 1.4423 & 1.4943 & 1.4611 \\
$\mathrm{C}(1)-\mathrm{C}\left(2^{\prime}\right)$ & 1.4424 & 1.4943 & 1.4611 \\
$\mathrm{C}(2)-\mathrm{C}(3)$ & 1.4298 & 1.4212 & 1.4523 \\
$\mathrm{C}\left(2^{\prime}\right)-\mathrm{C}(3)$ & 1.4298 & 1.4212 & 1.4523 \\
$\mathrm{C}(3)-\mathrm{C}(4)$ & 1.4176 & 1.4230 & 1.4165 \\
$\mathrm{C}\left(3^{\prime}\right)-\mathrm{C}(4)$ & 1.4175 & 1.4230 & 1.4165 \\
\hline
\end{tabular}

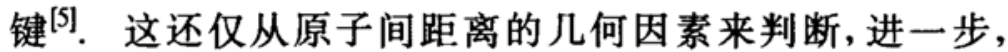
从原子电荷的分布变化更可看出 (见表 2), 自由基化后非常 有利于邻近羟基间形成分子内氢键 ${ }^{[5]}$. 现在都已知道, 氢键

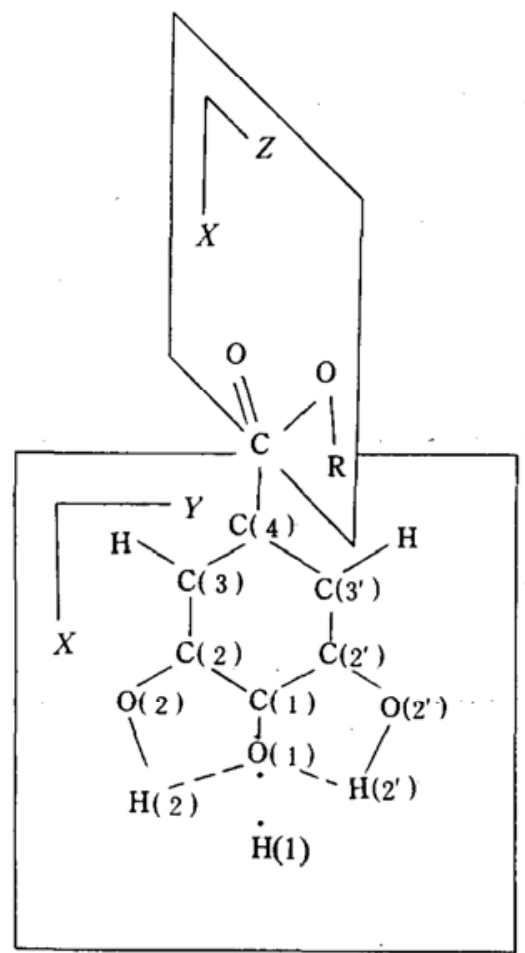

图 1 五倍子酸和酯及其自由基的 结构示意图

是一种特殊的带异性电荷的原子之间的相互作用 ${ }^{[6,7]}$. 两者距离愈短, 荷电量愈大, 氢键作用 就愈强. 联同 $C(1), C(2), C\left(2^{\prime}\right)$ 上原子电荷分布一起考察, 可以明显地看出, 五倍子酸及其酯 自由基化后极有利于 $\mathrm{C}_{2} \mathrm{O}_{2} \mathrm{H}$ 五元环的形成, 尤其是羧基对位的羟基失氢生成的自由基(参考 图1).

表 2 五倍子酸及其酯自由基化前后有关原子电荷的变化 $(|\mathrm{e}|)$

\begin{tabular}{lccc}
\hline \multicolumn{1}{c}{ 原 } & 分子的 & 对位自由基的 & 间位自由基的 \\
\hline $\mathrm{H}(1)$ & 0.1954 & - & 0.2236 \\
$\mathrm{H}(2)$ & 0.2056 & 0.2162 & - \\
$\mathrm{H}\left(2^{\prime}\right)$ & 0.2062 & 0.2158 & 0.2272 \\
$\mathrm{O}(1)$ & -0.2773 & -0.2941 & -0.2377 \\
$\mathrm{O}(2)$ & -0.2409 & -0.2140 & -0.2581 \\
$\mathrm{O}\left(2^{\prime}\right)$ & -0.2397 & -0.2135 & -0.2194 \\
$\mathrm{C}(1)$ & -0.0209 & 0.2322 & -0.0201 \\
$\mathrm{C}(2)$ & 0.0818 & 0.0114 & 0.1797 \\
$\mathrm{C}\left(2^{\prime}\right)$ & 0.0835 & 0.0114 & 0.0735 \\
\hline
\end{tabular}

更有趣地看到, 五倍子酸及其酯对位羟基失氢生成的自由基中 $\mathrm{O}(1)$ 的自旋密度显著升 高, 并且还与 $\mathrm{H}(2)$ 及 $\mathrm{H}\left(2^{\prime}\right)$ 的自旋密度反号 (表 3). 从成键电子配对自旋反号相关的角度 看, 这又进一步加强氢键并五元环的生成.

总结上述计算结果的数据并分析看, 由于自由基化后: (1) 原子间距离的变化, (2) 原子的 电荷分布及 (3) 原子的自旋密度, 都有利于 $\mathrm{O}-\mathrm{H} \cdots \mathrm{O}$ 氢键及 $\mathrm{C}_{2} \mathrm{O}_{2} \mathrm{H}$ 五元环的生成, 而两个 
表 3 五倍子酸及其酯自由基化前后有关原子的 $(\mathrm{S}+\mathrm{Pz})$ 的电子自旋密度的变化 $(\alpha=+; \beta=-)$

\begin{tabular}{lrcc}
\hline \multicolumn{1}{c}{ 原 } & 分子的 & 对位自由基的 & 间位自由基的 \\
\hline $\mathrm{H}(1)$ & -0.0237 & - & -0.0011 \\
$\mathrm{H}(2)$ & 0.0036 & -0.0016 & - \\
$\mathrm{H}\left(2^{\prime}\right)$ & 0.0026 & -0.0015 & 0.0006 \\
$\mathrm{O}(1)$ & -0.0036 & 0.3109 & 0.1102 \\
$\mathrm{O}(2)$ & 0.0600 & 0.1180 & 0.5016 \\
$\mathrm{O}\left(2^{\prime}\right)$ & 0.4912 & 0.1179 & -0.1137 \\
\hline
\end{tabular}

五元环又能与原来的苯环形成稠环共轭体系, 所以五倍子酸及其酯中羧基对位的羟基最易失 氢形成自由基, 并且能稳定地存在.

表 4 五倍子酸及其酯自由基化前后各分子的生成热 $(\times 4.1840 \mathrm{~kJ} / \mathrm{mol})$

\begin{tabular}{llcc}
\hline 分 & 分子的 & 对位自由基的 & 间位自由基的 \\
\hline GA & -212.01 & -193.49 & -173.09 \\
MG & -200.67 & -182.02 & -173.07 \\
EG & -204.72 & -186.06 & -175.70 \\
PG & -207.83 & -189.15 & $-\mathrm{i} 80.34$ \\
iso-PG & -206.01 & -187.54 & -178.61 \\
BG & -210.70 & -192.08 & -183.24 \\
t-BG & -204.91 & -186.22 & -177.79 \\
\hline
\end{tabular}

上面关于原子间距, 原子电荷及原子自旋密度的分析都是微观机理, 而生成热则是分子体系 的稳定性的宏观综合量度, 前者是后者的原因, 而后者是前者的效果. 表 4 给出五倍子酸及其 酯自由基化前后生成热的变化, 它从能量观点给出了上面的结论. 从表 4 明显看出, 羭基对位 的羟基失氢生成的自由基要比羭基间位的羟基失氢生成的自由基稳定约 $(10-20) \times 4.1840 \mathrm{~kJ} / \mathrm{mol}$, 这是一个相当不小的热稳定性.

\section{参考文甫}

[1] Uchida, S., Edamatso, R., Hiramatso, M. et al., Med. Sci. Res., 1987, 15:831.

[2] Dziedzic, C. Z., Hudson, B. J. F., Food Chem., 1981, 14:45.

[3] Maddox, D. N., Int. Flavours Food Addit., 1976, (7): 117.

[4] Pongracz, G., Int. J. Vitam. Nutr. Res., 1973, 43:517.

[5] 徐光宪, 物质结构, 高等教育出版社, 1959.

[6] Williams, D. E., Yan, J. M., Advances in Atomic and Molecular Physics, 1987, 23:87.

[7] Kong, J., Yan, J. M., Int. J. Quantum Chem., 1993, 46: 239. 